\title{
Os resíduos de serviço de saúde e seus impactos ambientais: uma revisão bibliográfica
}

\author{
Residues of health services and their environmental impacts: a bibliographical \\ revision
Les résidus de service de santé et ses impacts environnementaux: une révision bibliographique

Los residuos de servicio de salud y sus impactos ambientales: una revisión bibliográfica

\author{
Vera Araujo Cafure* \\ (vcafure@hotmail.com) \\ Suelen Regina Patriarcha-Graciolli**
(suelenpatriarcha@yahoo.com.br)
}

Recebido em 23/02/2014; revisado e aprovado em 24/07/2014; aceito em 22/11/2014

DOI: http:/ / dx.doi.org/10.1590/151870122015206

\begin{abstract}
Resumo: As normas brasileiras de Resíduos de Serviços de Saúde (RSS) definem o descarte dos resíduos de serviços de saúde. Com o objetivo de discorrer sobre os RSS e seus impactos ambientais, foi realizada uma pesquisa bibliográfica. Doze artigos foram encontrados, e observou-se uma discreta elevação dos artigos publicados entres os anos de 2005 e 2011. Na região sul, encontrou-se a maior concentração de pesquisas relacionadas ao tema, e também as maiores taxas de processamentos desses resíduos. Percebeu-se a necessidade de desenvolvimento de ações com os órgãos geradores dos RSS, já que existe um distanciamento entre a teoria e o que é realizado nos estabelecimentos de saúde. Palavras-chave: RSS. Meio Ambiente. Materiais infectantes.
\end{abstract}

Abstract: Brazilian norms of Residues of Health Services (RHS) define the discard of residues of health services. With the purpose of discoursing about RHS and their environmental impacts a bibliographical research was accomplished. Twelve articles were found and it was perceived a discreet increment of articles published between the years of 2005 and 2011. In the south area, it was found the greatest concentration of researches related to the theme and, also, the largest taxes of processing those residues. It was noticed the need of developing actions with the producing entities of RHS, considering that exists a big distance between the theory and what it is really performed on health institutions.

Key words: RHS. Environment. Infectious Materials.

Résumé: Les réglementations brésiliennes sur les Résidus de Services de Santé (RSS) définent la décharge des résidus de santé. Une recherche bibliographique a été réalisée en objectivant le discours sur les RSS et ses impacts environnementaux. Dans les douze articles retrouvés s' observe une discrète augmentation des articles publiés entre les années 2005 et 2011. Dans la région Sud se trouve la plus grande concentration de recherches concernantes à ce sujet et aussi les plus grands taux de traitement de ces résidus. La necessité de développement des actions avec les organismes produisants des RSS a été apperçue, une fois qu'il existe un éloignement entre la théorie et ce qui est pratiqué dans les établissements de santé

Mots-clés: RSS. Environnement. Matériaux infectants.

Resúmen: Las normas brasileñas de Residuos de Servicios de Salud (RSS) definen el desecho de los residuos de servicios de salud. Con el objetivo de discurrir sobre los RSS y sus impactos ambientales se realizó una investigación bibliográfica. Se encontraron doce artículos y se observó un aumento discreto de los artículos publicados entre los años de 2005 y 2011. En la región sur, se encontró la mayor concentración de investigaciones relacionadas con el tema y, también, las mayores tasas de procesamiento de dichos residuos. Se evidenció la necesidad de desarrollar acciones con los órganos generadores de RSS, ya que existe un distanciamiento entre la teoría y lo que es realizado en los establecimientos de salud.

Palabras clave: RSS. Medio ambiente. Materiales infecciosos.

\section{Introdução}

Segundo a norma brasileira NBR 10004/04 da Associação Brasileira de Normas Técnicas (ABNT), resíduos sólidos são definidos como resíduos em estado sólido e semissólidos, produtos da ação da indústria, de atividades domésticas, do comércio, de serviços agrícolas e também de serviços hospitalares (ABNT, 2004).

Os Resíduos Sólidos Hospitalares ou, como é mais comumente denominado, lixo hospitalar, podem tornar-se um problema de saúde pública, devido principalmente à

\footnotetext{
* UNIGRAN CAPITAL, Campo Grande, Mato Grosso do Sul, Brasil.

** Universidade Católica Dom Bosco (UCDB) e UNIGRAN CAPITAL. Campo Grande, Mato Grosso do Sul, Brasil.
} 
falta de informações da população sobre suas particularidades, ocasionando riscos à saúde.

Resíduos de Serviços da Saúde (RSS),

para as normas brasileiras de terminologia, classificação, manuseio e coleta de resíduos e serviços de saúde, são definidos como resíduos resultantes das atividades exercidas por estabelecimentos prestadores de serviços de saúde, abrangendo os resíduos provenientes das mais diversas fontes potencialmente geradoras, como hospitais, clínicas médicas, veterinárias, odontológicas, farmácias, ambulatórios, postos de saúde, laboratório de análises clínicas, laboratórios de análises de alimento, laboratórios de pesquisa, consultórios médicos e odontológicos, empresas de biotecnologia, casas de repouso e casas funerárias (SCHNEIDER, 2004).

Segundo Ramos et al. (2011), os RSS compõem parte importante do total dos Resíduos Sólidos Urbanos (RSU) não pela quantidade gerada, mas pelo potencial risco que afeta à saúde ambiental e coletiva.

Para Schneider (2004), o risco ambiental é o risco que ocorre no meio ambiente e pode ser submetido à classificação de acordo com o tipo de atividade; exposição instantânea, crônica; probabilidade de ocorrência; severidade; reversibilidade; visibilidade; duração e possibilidade de ocorrência de seus efeitos em vários locais ao mesmo tempo. No contexto da gestão governamental, o risco ambiental pode ser classificado como de saúde pública, recursos naturais, desastre natural e introdução de novos produtos.

De acordo com Brasil (2001a), os RSS representam uma fonte de risco à saúde e ao meio ambiente principalmente pela falta de adoção de procedimentos técnicos adequados no manejo dos diferentes resíduos, como material biológico contaminado, objetos perfurocortantes, peças anatômicas, substâncias tóxicas, inflamáveis e radiativas.

Existe também risco às pessoas que manuseiam os RSS dentro e fora dos estabelecimentos geradores. Há ainda os riscos que podem afetar a comunidade hospitalar, principalmente o grupo constituído por pacientes em tratamento que, devido ao estado de doença, encontra-se com suas defesas comprometidas. O manejo inadequado dos RSS pode causar risco ambiental, que ultrapassam limites do estabelecimento, podendo gerar doenças e ainda perda da qualidade de vida da população que, direta ou indiretamente, venha ter contato com o material descartado, no momento do seu transporte para fora do estabelecimento e seu tratamento e destinação (BRASIL, 2001a).

Em 07 de dezembro de 2004, a Agência Nacional de Vigilância Sanitária (ANVISA) dispôs a Resolução da Diretoria Colegiada (RDC) $n^{\circ} 306$, que regulamenta normas para gestão de resíduos de serviços de saúde, portos, aeroportos, e terminais rodoviários formados por representantes do governo federal, autoridade normativa para discutir, elaborar regulamentos específicos ao meio ambiente (BRASIL, 2004). Salienta-se ainda que, na década de 1970, foi criado o Conselho Nacional de Meio Ambiente (CONAMA) o qual, em 29 de abril de 2005, na Resolução $n^{0} 358$, dispõe sobre tratamento e disposição final dos resíduos de saúde (BRASIL, 2005).

Com a Política Nacional de Resíduo Sólido (PNRS), por meio da Lei 12.305, que dispõe diretrizes aplicáveis aos resíduos sólidos, estabeleceu-se proteção da saúde e qualidade ao meio ambiente (BRASIL, 2010).

"Os RSS são de natureza heterogênea" (GARCIA; ZANETTI-RAMOS, 2004, p. 746), e diferentes classificações já foram propostas por várias entidades, como o CONAMA, a ANVISA e ainda governos estaduais e municipais, já que se faz necessária uma classificação e separação desses tipos de resíduos, além da preocupação com os manipuladores desse material e com o meio ambiente.

Há de se evidenciar que existe um amparo legal, composto por leis e normas para a minimização dos problemas envolvendo as gestões dos resíduos sólidos de saúde. Entretanto é constatado que, na prática, ainda há um não cumprimento das normas vigentes, pois são observados que a cada dia são desprezados de maneira inadequada os resíduos sólidos hospitalares de área específica, como centro cirúrgico, onde restos de tecido humano, placenta, resíduos pós-aborto, bolsa de hemoderivados e prótese, são descartadas em lixeira comum hospitalar sem a menor preocupação dos impactos que estes podem acarretar no ambiente e no ser humano assim como seu destino final.

Assim, esta pesquisa busca realizar um estudo de revisão bibliográfica, para investi- 
gar se as normas existentes para o descarte desses resíduos estão sendo cumpridas pelos profissionais da saúde e também pelas empresas às quais eles estão ligados profissionalmente; e ainda, entender o impacto que o ambiente e a sociedade como um todo recebem quando não há um descarte correto desses resíduos sólidos hospitalares.

\section{Material e métodos}

Para o presente trabalho realizou-se uma pesquisa bibliográfica. Segundo Almeida (2011), a pesquisa bibliográfica busca relações entre conceitos, características e ideias, muitas vezes unindo dois ou mais temas.

Para Severino (2007), essa modalidade de pesquisa se caracteriza a partir do registro disponível, que decorre de pesquisas já realizadas, em livros, artigos, teses e documentos impressos. Dessa forma, os textos tornam-se fontes dos temas que serão trabalhados e pesquisados. Para Martins e Lintz (2000), essa pesquisa busca conhecer e analisar contribuições científicas sobre determinado assunto. Alves (2007, p. 55) escreve

Pesquisa bibliográfica é aquela desenvolvida exclusivamente a partir de fontes já elaboradas - livros, artigos científicos, publicações periódicas, as chamadas fontes de "papel". Tem como vantagem cobrir uma ampla gama de fenômeno que o pesquisador não poderia contemplar diretamente.
Para a busca de trabalhos como fontes de pesquisa, foram empregados os descritores: lixo hospitalar; resíduos hospitalares; e resíduos em saúde; na base de dados Biblioteca Virtual em Saúde (BVS), no período de fevereiro a abril de 2013.

A questão que orientou a busca pelos artigos nessa revisão foi: $\mathrm{Na}$ literatura científica nacional, como tem sido relatado o descarte e manuseio, assim como os impactos ao meio ambiente dos RSS?

Na divisão da Ciência da Saúde da BVS encontraram-se 566 artigos, nos idiomas português, inglês e outros. Dentre esse montante, somente 44 artigos continham textos completos liberados para consulta e em português.

Dessa forma, após um estudo dos 44 artigos, foram selecionados 12 que enfatizaram o descarte dos resíduos sólidos em saúde.

A análise das informações foi realizada por meio de leitura exploratória do material encontrado, em uma abordagem qualitativa.

\section{Resultados e discussão}

Após a busca dos artigos que correspondessem ao tema pesquisado, excluindo-se os artigos que se tratavam de revisão de literatura, foram obtidos 12 artigos, dispostos entre os anos de 2005 e 2011. Uma discreta evolução no número de artigos publicados ao longo desses anos, sobre o tema estudado aqui, foi possível de ser observado, conforme gráfico 1. 


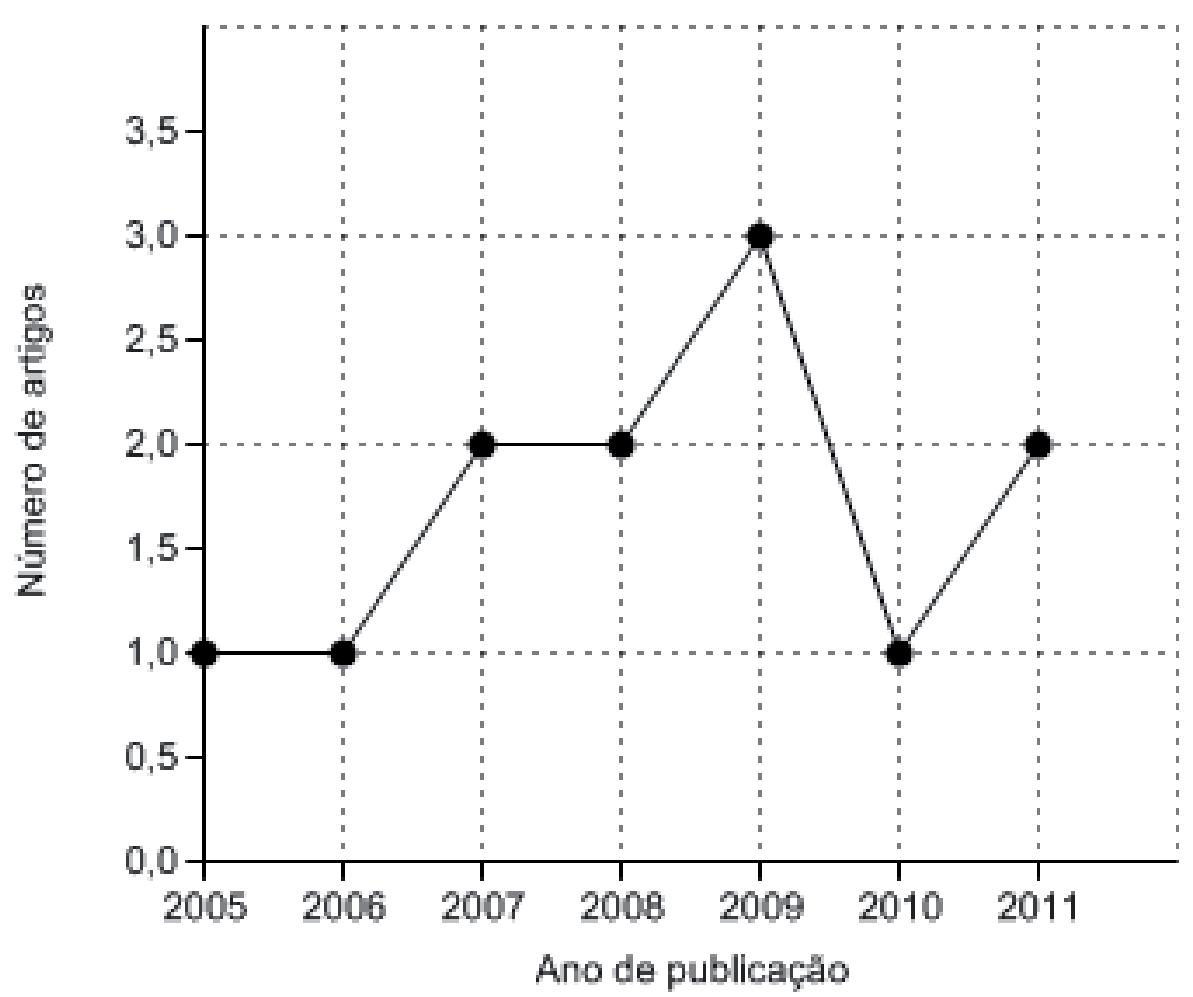

Gráfico 1 - Evolução do número de artigos publicados entre os anos de 2005 e 2011 sobre RSS.

A partir do exposto, foi possível verificar uma discreta evolução no número de artigos publicados sobre RSS entre os anos de 2005 e 2011, já que, durante os anos de 2005 e 2006, o número de artigos que abordaram esse tema, encontrados na base BVS, foi de apenas um para cada ano. Já para os anos de 2007, 2008, pôde-se observar que o número dobrou em relação aos dois anos anteriores. Em 2009, foi encontrado o triplo de artigo que no ano de 2005. No ano de 2010, o número volta a cair para apenas um trabalho e, em 2011, a pesquisa mostrou que as publicações voltaram a crescer. Cabe ressaltar que as pesquisas utilizadas neste estudo não abordaram os trabalhos de revisão de literatura. Caso esses trabalhos tivessem sido incluídos, o número de trabalhos seria ainda maior.

O sensível crescimento no número de artigos publicados nesse período e relacionado ao tema RSS tem grande relação com a recente preocupação com as questões ambientais. Foi em 2005, por meio da resolução $\mathrm{n}^{\circ} 358$ (BRASIL, 2005), que o CONAMA dispôs sobre a destinação e tratamento de RSS. Anteriormente, já havia outras Resoluções do CONAMA sobre o assunto, como a n 5 de 1993 (BRASIL, 1993) e a 283 de 2001 (BRASIL, 2001b), no entanto essas Resoluções não abordavam o tratamento desse tipo de resíduo, apenas os classificava e propunha a adoção de medidas para controle e disposição desse material, ficando a resolução $\mathrm{n}^{\circ} 5$ revogada, e a 283 complementada pela de $\mathrm{n}^{\circ}$ 358 (BRASIL, 2005).

Para melhor disposição e compreensão dos estudos encontrados para composição do material bibliográfico desta pesquisa, segue quadro (Quadro 1), o qual foi construído a partir das datas de publicação, do mais antigo ao mais recente, dos artigos que compõem a base deste estudo. 


\begin{tabular}{|c|c|c|c|c|}
\hline & Ano & Título do Artigo & Autor (es) & Periódico \\
\hline 1. & 2005 & $\begin{array}{l}\text { Diagnóstico dos resíduos de serviços de } \\
\text { saúde no interior do Rio Grande do Sul }\end{array}$ & $\begin{array}{l}\text { SILVA, C. E. da; } \\
\text { HOPPE, A. E. }\end{array}$ & $\begin{array}{l}\text { Eng. sanit. ambient., v. 10, } \\
\text { n. } 2, \text { p. } 146-151 .\end{array}$ \\
\hline 2. & 2006 & $\begin{array}{c}\text { Diagnóstico do problema dos resíduos } \\
\text { sólidos hospitalares: o caso de Campina } \\
\text { Grande }(\mathrm{PB})\end{array}$ & $\begin{array}{l}\text { VASCONCELLOS, E. } \\
\text { A. de et al. }\end{array}$ & Hygeia, v. 2, n. 3, p. 28-34. \\
\hline 3. & 2007 & $\begin{array}{c}\text { A situação dos hospitais quanto ao } \\
\text { gerenciamento dos aspectos e impactos } \\
\text { ambientais. }\end{array}$ & $\begin{array}{l}\text { PFITSCHER, E. D. } \\
\text { et al. }\end{array}$ & $\begin{array}{l}\text { Cadernos EBAPE.BR, v. 5, } \\
\text { n. } 3 .\end{array}$ \\
\hline 4. & 2007 & $\begin{array}{l}\text { O processo de formação em saúde: o } \\
\text { saber resíduos sólidos de serviços de } \\
\text { saúde em vivências práticas }\end{array}$ & $\begin{array}{l}\text { CORRÊEA. L. B.; } \\
\text { LUNARDI, V. L.; } \\
\text { CONTO, S. M. de. }\end{array}$ & $\begin{array}{l}\text { Rev Bras Enferm, v. 60, n. } \\
\text { 1, p. 21-25. }\end{array}$ \\
\hline 5. & 2008 & $\begin{array}{l}\text { Construção do saber sobre resíduos } \\
\text { sólidos de serviços de saúde na } \\
\text { formação em saúde }\end{array}$ & $\begin{array}{l}\text { CORRÊA, L. B.; } \\
\text { LUNARDI, V. L.; } \\
\text { SANTOS, S. S. C. }\end{array}$ & $\begin{array}{l}\text { Rev. Gaúcha Enfermagem, } \\
\text { v. } 29, \text { n. } 4 \text {, p. 557-564. }\end{array}$ \\
\hline 6. & 2008 & $\begin{array}{c}\text { Avaliação do Sistema de Gestão } \\
\text { dos resíduos sólidos do Hospital de } \\
\text { Clínicas de Porto Alegre }\end{array}$ & $\begin{array}{l}\text { NAIME, R.; } \\
\text { RAMALHO, A. H. } \\
\text { P.; NAIME, I. S. }\end{array}$ & $\begin{array}{l}\text { Revista Espaço para a } \\
\text { Saúde, v. 9, n. 1, p. 1-17. }\end{array}$ \\
\hline 7. & 2009 & $\begin{array}{l}\text { Um olhar sobre a interface trabalho } \\
\text { hospitalar e os problemas ambientais }\end{array}$ & $\begin{array}{l}\text { CAMPONOGARA, } \\
\text { S.; RAMOS, F. R. S.; } \\
\text { KIRCHHOF, A. L. C. }\end{array}$ & $\begin{array}{l}\text { Rev Gaúcha Enferm., v. 30, } \\
\text { n. 4, p. 724-731. }\end{array}$ \\
\hline 8. & 2009 & $\begin{array}{l}\text { Gerenciamento dos resíduos sólidos } \\
\text { dos serviços de saúde: aspectos do } \\
\text { manejo interno no município de } \\
\text { Marituba, Pará, Brasil. }\end{array}$ & $\begin{array}{l}\text { SALES, C. C. de L. } \\
\text { et al. }\end{array}$ & $\begin{array}{l}\text { Ciência E Saúde Coletiva, v. } \\
\text { 14, n. 6, p. 2231-2238. }\end{array}$ \\
\hline 9. & 2009 & $\begin{array}{r}\text { Resíduos Sólido } \\
\text { um estudo sol }\end{array}$ & $\begin{array}{l}\text { CAMARGO, M. E. } \\
\text { et al. }\end{array}$ & $\begin{array}{l}\text { Scientia Plena, v. 5, n. 7, p. } \\
\text { 1-14. }\end{array}$ \\
\hline 10. & 2010 & $\begin{array}{l}\text { Análise preliminar de riscos sobre o } \\
\text { gerenciamento dos resíduos de serviços } \\
\text { de saúde de uma instituição de ensino } \\
\text { em Mato Grosso do Sul: estudo de caso }\end{array}$ & $\begin{array}{l}\text { SHINZATO, M. P. } \\
\text { et al. }\end{array}$ & $\begin{array}{l}\text { Rev. Bras. Saúde Ocup., } \\
\text { v. 35, n. 122, p. 340-352. }\end{array}$ \\
\hline 11. & 2011 & $\begin{array}{l}\text { Vulnerabilidade no manejo dos } \\
\text { resíduos de serviços de saúde de João } \\
\text { Pessoa (PB, Brasil) }\end{array}$ & RAMOS, Y S. et al. & $\begin{array}{l}\text { Ciência E Saúde Coletiva, } \\
\text { v. 16, n. 8, p. 3553-3560. }\end{array}$ \\
\hline 12. & 2011 & $\begin{array}{l}\text { Resíduos Sólidos de Serviço de Saúde: } \\
\text { uma fotografia do comportamento da } \\
\text { equipe de enfermagem }\end{array}$ & $\begin{array}{l}\text { DOI, K. M.; MOURA, } \\
\text { G. M. S. S. de }\end{array}$ & $\begin{array}{l}\text { Rev. Gaúcha Enfermagem, } \\
\text { v. 32, n. 2, p. 338-344. }\end{array}$ \\
\hline
\end{tabular}

Quadro 1 - Lista de artigos selecionados na base Biblioteca Virtual em Saúde (BVS) de 2005 a 2011.

Foi possível observar, após a leitura e análise dos artigos encontrados, que os autores Silva e Hoppe (2005), Vasconcellos et al. (2006), Pfitscher et al. (2007), Naime, Ramalho e Naime (2008), Camponogara, Ramos e Kirchhof (2009); Camargo et al. (2009), Shinzato et al. (2010) e Ramos et al.(2011) buscaram dialogar sobre o manejo dos resíduos sólidos hospitalares pelos profissionais ligados à área de saúde.

Ramos et al. (2011) tiveram como foco da pesquisa a vulnerabilidade existente no manejo dos resíduos de serviços de saúde de João Pessoa, PB, no Brasil. Os autores construíram um checklist, com o intuito de descobrir a origem dos problemas e definir ações corretivas para o manejo ambientalmente correto dos RSS. Tiveram como referencial os requisitos para o gerenciamento de RSS da RDC $\mathrm{n}^{\circ} 306$ (BRASIL, 2004).

Estes instrumentos nortearam a construção de sete parâmetros de vulnerabilidade no manejo dos RSS: segregação, tratamento prévio, acondicionamento, transporte interno, armazenamento intermediário, armazenamento externo e disposição final de estabelecimentos de saúde de atenção primária, secundária e terciária, localizados em João Pessoa. (RAMOS et al., 2011, p. 3555).

Foi possível observar que a vulnerabilidade do manejo dos RSS do município de João Pessoa/PB, de 48,02\%, é bastante significativa, traduzindo a não observância dos elementos legais e tático-operacionais do processo. Assim, o autor conclui que a educação é uma maneira de dar sentido para 
as práticas do dia a dia, pois é a partir da vida cotidiana, das necessidades e interesses das pessoas que as exigências da sociedade do planeta precisam ser trabalhadas.

Em outra região do país investigada, a região sul, a referida vulnerabilidade também foi demarcada por Camponogara, Ramos e Kirchhof (2009), os quais enfatizam que os sujeitos, embora expressem certa preocupação com os problemas ambientais e desenvolvam algumas ações em prol da preservação ambiental em âmbito doméstico, parecem não transferir esse conhecimento para o seu cotidiano no local de trabalho.

Podemos destacar também a dificuldade manifestada pelos trabalhadores em listar outros fatores de relação entre o hospital e o meio ambiente. Para muitos a pergunta era impactante, deixando explícito o fato de que trabalhador é fortemente afetado pela norma institucional e têm dificuldades de argumentar, de forma crítica, sobre a possibilidade de outras questões relacionadas ao trabalho hospitalar. (CAMPONOGARA; RAMOS; KIRCHHOF, 2009, p. 729).

Para Naime, Ramalho e Naime (2008), a falta de informações sobre o assunto é um dos principais motivos para a ausência de projetos bem sustentados que determinem melhorias no setor. Particularmente os resíduos dos serviços de saúde merecem atenção especial em suas fases de separação, acondicionamento, armazenamento, coleta, transporte, tratamento e disposição final.

Corrêa, Lunardi e Conto (2007) assim como Corrêa, Lunardi e Santos (2008), enfatizaram a necessidade de formação dos profissionais da área da saúde menos fragmentada quando se trata de RSS, já que realizaram pesquisas em instituições de ensino superior e puderam concluir que grande parte dos profissionais, apesar de conhecerem, não realiza procedimentos adequados quanto ao condicionamento, armazenamento e também o descarte de RSS.

Doi e Moura (2011) investigaram a percepção de profissionais da área da saúde quanto aos RSS. As autoras encontraram uma maioria de profissionais dizendo que realizam a separação dos RSS. Os profissionais que relataram não realizar tal procedimento justificaram suas ações; alegaram falta de tempo, já que o número de profissionais da unidade é inadequado e a prioridade é o cuidado com o paciente.

Os impactos ambientais causados pelo gerenciamento inadequado dos resíduos hospitalares podem atingir grandes proporções, levando a contaminações e elevados índices de infecção hospitalar, ou até mesmo à geração de epidemias devido a contaminações do lençol freático pelos diversos tipos de resíduos dos serviços de saúde.

Para que fosse possível a visualização das principais regiões em que o tema tem tido maior destaque, as pesquisas levantadas por este estudo também foram agrupadas por regiões brasileiras, conforme o gráfico 2 . É importante ressaltar que as pesquisas foram agrupadas por regiões de estudo, e não quanto ao local do periódico de publicação. 


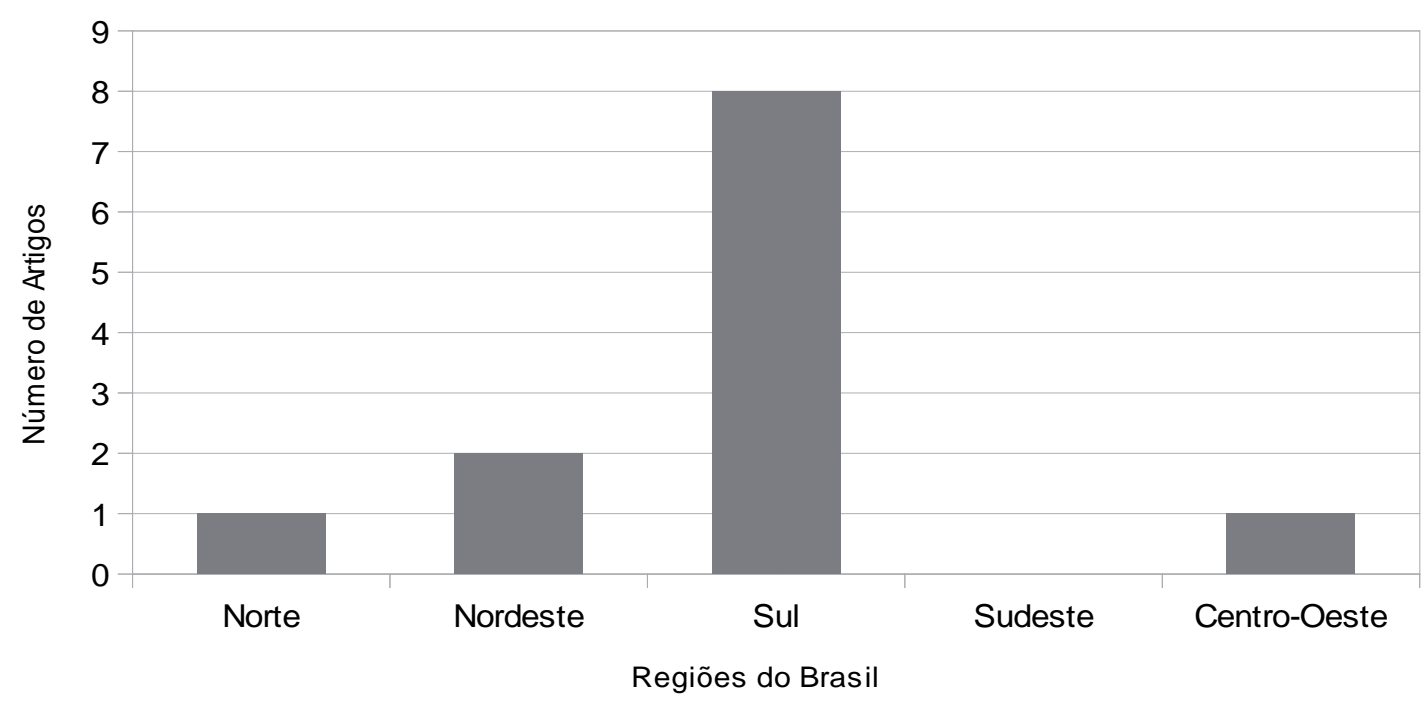

Gráfico 2 - Regiões do Brasil em que foram encontrados artigos sobre o tema RSS, na base de dados BVS entre os anos de 2005 e 2011.

De acordo com informações levantadas por meio do estudo dos artigos pesquisados por regiões, evidenciou-se que, na Região Sul, concentra-se um maior número de pesquisa relacionada aos resíduos sólidos de saúde, um total de oito artigos publicados.

A região Nordeste vem em seguida, com a presença de dois artigos publicados, e as regiões Norte e Centro-Oeste, apresentaram-se um artigo cada.

Dados do IBGE demonstram que a região Sul do Brasil apresenta uma das maiores taxas de processamento correto dos RSS nos seus municípios. Nessa região, de um total de 997 municípios que contam com coleta ou recebimento de RSS, 705 declaram processar os RSS, sendo a incineração e o tratamento em autoclave a maioria absoluta desses processamentos. $\mathrm{O}$ quadro 2 apresenta o total de municípios de cada região do Brasil agrupando-os quanto à existência ou não de processamento de RSS, e o quadro 3 apresenta os dados referentes ao processamento dos RSS distribuídos por região, segundo dados apresentados pela Pesquisa Nacional de Saneamento Básico 2008, do IBGE (IBGE, 2010).

\begin{tabular}{|l|c|c|c|}
\hline \multicolumn{1}{|c|}{ Região do Brasil } & $\begin{array}{c}\text { Total de } \\
\text { Municípios }\end{array}$ & $\begin{array}{c}\text { Existência de } \\
\text { processamento de RSS }\end{array}$ & $\begin{array}{c}\text { Não existe processamento } \\
\text { de RSS }\end{array}$ \\
\hline Norte & 304 & 140 & 164 \\
\hline Nordeste & 1309 & 790 & 519 \\
\hline Sul & 997 & 705 & 292 \\
\hline Sudeste & 1492 & 806 & 686 \\
\hline Centro-Oeste & 367 & 172 & 195 \\
\hline
\end{tabular}

Quadro 2 - Total de Municípios do Brasil separados por regiões que apresentam coleta e/ou recebimento de RSS.

\begin{tabular}{|c|c|c|c|c|c|c|}
\hline $\begin{array}{c}\text { Região do } \\
\text { Brasil }\end{array}$ & Incineração & $\begin{array}{c}\text { Queima em } \\
\text { forno simples }\end{array}$ & $\begin{array}{c}\text { Queima a } \\
\text { céu aberto }\end{array}$ & $\begin{array}{c}\text { Tratamento } \\
\text { em Autoclave }\end{array}$ & $\begin{array}{c}\text { Tratamento por } \\
\text { micro-ondas }\end{array}$ & Outro \\
\hline Norte & 48 & 10 & 69 & 1 & - & 23 \\
\hline Nordeste & 276 & 48 & 439 & 6 & - & 75 \\
\hline Sul & 487 & 5 & 1 & 461 & 19 & 67 \\
\hline Sudeste & 488 & 38 & 68 & 285 & 57 & 99 \\
\hline $\begin{array}{c}\text { Centro- } \\
\text { Oeste }\end{array}$ & 80 & 30 & 39 & 10 & - & 27 \\
\hline
\end{tabular}

Quadro 3 - Tipos de processamento dos RSS nos municípios das regiões do Brasil. 
Diante dos dados apresentados, podese observar que, na região Sul, das cidades que têm coleta ou recebimento de RSS, aproximadamente $70 \%$ declaram possuir e seguir processamento para os RSS (IBGE, 2010). Nesse caso, torna-se bastante justificável o fato de, nesta pesquisa, o número de artigos encontrados que fazem referência aos RSS na região Sul, ter sido bastante significativo, se comparado às demais regiões, já que os dados demonstram que a região tem se preocupado com a destinação desse tipo de resíduo.

A Região Nordeste vem em seguida com um número expressivo, se comparado com as demais regiões do país, de aproximadamente $60 \%$ de seus RSS seguindo algum tipo de processamento (IBGE, 2010). No entanto o processamento mais recorrente entre as cidades da região Nordeste é a queima de RSS a céu aberto, causando outros problemas ambientais, como a poluição do ar e aumento do efeito estufa.

As regiões Norte e Centro-Oeste apresentam índices parecidos quanto à existência de processamento dos RSS, em torno de $46 \%$ (IBGE, 2010). Paralelamente a esse dado, podemos destacar que, nessas duas regiões, o número de artigos encontrados com o tema RSS foi apenas um, o que também demonstra que as pesquisas em RSS não têm sido muito significativas, assim como as políticas de implantação de métodos eficientes e de acordo com a Resolução n ${ }^{\circ} 358$ do CONAMA.

Por fim, a região Sudeste, aparece, segundo o IBGE (2010), com 54\% dos seus municípios com algum processamento dos RSS. Apesar de, nesta pesquisa, não terem sido encontrados artigos com o tema RSS nessa região, os processamentos mais comuns nessas áreas são a incineração e o tratamento em autoclave.

Diante do exposto, iniciou-se uma busca a partir da legislação vigente para a destinação correta dos resíduos sólidos hospitalares. A Portaria MINTER 53, de $1^{\circ}$ de março de 1979 (BRASIL, 1979), foi a primeira legislação federal que abordou os resíduos hospitalares e indicou a obrigatoriedade de incinerá-los. Essa portaria foi alterada pela resolução CONAMA n ${ }^{\circ} 6$ em 1991 (BRASIL, 1991), que desobrigou a incineração ou qualquer outro tratamento de queima dos resíduos sólidos provenientes dos estabelecimentos de saúde. Em 1993, esses documentos foram revogados pela Resolução $n^{\circ} 5$ (BRASIL, 1993), que, mais tarde, também foi revogada pela Resolução 358 (BRASIL, 2005), vigente até os dias atuais.

Vale destacar que, em 1993, por meio da Resolução CONAMA no 5 , já se definiam os procedimentos mínimos para o gerenciamento de resíduos sólidos provenientes de serviços de saúde, portos e aeroportos: Resíduos Sólidos, Plano de Gerenciamento de Resíduos Sólidos, Sistema de Tratamento de Resíduos Sólidos e Sistema de Disposição Final de Resíduos Sólidos (BRASIL, 1993).

A ABNT publicou a NBR 10.004, em maio de 2004, que estabelece os critérios de classificação e os códigos para a identificação dos resíduos de acordo com suas características (ABNT, 2004).

No âmbito da ABNT, existem algumas normas relativas ao controle dos RSS, que podem ser observadas, conforme quadro 4 .

\begin{tabular}{|l|l|}
\hline \multicolumn{1}{|c|}{ NORMAS } & \multicolumn{1}{c|}{ DESCRIÇÃO } \\
\hline NBR 10.004 maio/2004 & $\begin{array}{l}\text { Classifica os Resíduos Sólidos quanto aos riscos potenciais ao meio ambiente } \\
\text { e à saúde pública. }\end{array}$ \\
\hline NBR 12.807 jun/2013 & Terminologia dos Resíduos de Serviços de Saúde. \\
\hline NBR 12.808 jan/1993 & Classificação de Resíduos de Serviços de Saúde - RSS, \\
\hline NBR 12.809 abr/2013 & Procedimento de Manuseio dos Resíduos de Serviços de Saúde. \\
\hline NBR 12.810 jan/1993 & Procedimento de Coleta de Resíduos de Serviços de Saúde \\
\hline NBR 7.500 mar/2000 & Símbolos de Risco e Manuseio para transporte Armazenagem de Materiais. \\
\hline NBR 9.190 maio/1994 & Classificação de sacos plásticos para acondicionamento de lixo. \\
\hline NBR 9.191 maio/2008 & Especificação de sacos plásticos para acondicionamento de lixo. \\
\hline
\end{tabular}

Quadro 4 - Normas relativas aos RSS (ABNT, 1993a; 1993b; 1994; 2000; 2004; 2008; 2013a; 2013b). 
Embora existam evidências de uma tomada de consciência dos problemas ambientais, o ser humano recebe forte influência do meio em que vive ao mesmo tempo em que o influencia em inúmeras situações, como na que concerne ao nível de higiene e de limpeza pública. Dessa forma, os RSS fazem parte do montante de resíduos sólidos gerados nos municípios e, embora representem uma pequena parcela, devem ser estudados com atenção devido ao grau de periculosidade a eles atribuídos (FERREIRA, 1995).

Para Ferrareze et al. (2005), a produção contínua e inesgotável de resíduos sólidos tem gerado constantes preocupações, já que os resíduos podem oferecer riscos, não só à sociedade, mas também ao meio ambiente. Para esses autores

[...] nas últimas décadas o gerenciamento de resíduos doméstico, industrial, bem como, aquele produzido em serviços de saúde tem envolvido estudiosos da área de saúde, ambientalistas, entre outros. Os esforços desses estudiosos são em prol de medidas eficazes de tratamento ou de destinação final desses resíduos. (FERRAREZE et al., 2005, p.134).

$O$ direito ambiental brasileiro engloba dois princípios básicos - o Princípio da Precaução e do Poluidor Pagador -, que, se fossem colocados em prática por parte da população em geral, poderiam atenuar os problemas de depósito de lixos, pois estes fazem referência ao fato da cautela que a população em geral deve ter em relação a qualquer tipo de resíduo e, ao ônus que deve ser suportado ao gerador do resíduo depositado em lugares indevidos (RIBEIRO, 2008).

A Constituição promulgada em 1988 em seu artigo 196 diz que "saúde é um direito de todos e dever do Estado". Para alcançar este objetivo fazem-se necessárias políticas econômicas e sociais que buscam o bem estar, $\mathrm{o}$ bem social e a redução do risco de doenças e de outros agravos, garantindo acesso às ações e serviços de saúde de maneira universal e igualitária. Ao longo dos tempos, as Instituições Hospitalares passaram de simples locais destinados ao atendimento e abrigo, a instituições de cura, transmissão e formação do saber. (CAMARGO et al., 2009, p. 1)

Na Lei 6.938 de 1981, o Art. 4º, VIII, retrata que os recursos ambientais são escassos, sua produção e consumo geram reflexos que podem resultar em degradação e escassez.
Além disso, ao utilizar gratuitamente um recurso ambiental, está se gerando um enriquecimento ilícito, pois, como o meio ambiente é um bem que pertence a todos, todos deveriam ter acesso e participação de forma igualitária a ele, no entanto parte da comunidade nem utiliza determinados recursos ou, se utilizam, o fazem em menor escala (BRASIL, 1981).

O Princípio do Usuário Pagador estabelece que quem utiliza o recurso ambiental deve suportar seus custos, sem que essa cobrança resulte na imposição taxas abusivas. Então, não há que se falar em Poder Público ou terceiros suportando esses custos, mas somente naqueles que dele se beneficiaram. Princípio do Poluidor Pagador obriga quem poluiu a pagar pela poluição causada ou que pode ser causada.

Os RSS, aqueles resultantes de atividades exercidas por prestadores de assistência médica, odontológica, laboratorial, farmacêutica e instituições de ensino e pesquisas médicas relacionadas tanto à saúde humana quanto veterinária, necessitam de processos diferenciados em seu manejo, exigindo ou não tratamento prévio à sua disposição final (SILVA; HOPE, 2005; BRASIL, 2005).

A Lei da Política Nacional de Resíduos Sólidos, Lei 12.305 de 2 de agosto de 2010, possui uma subseção dedicada especialmente aos resíduos de serviços de saúde, na qual define os estabelecimentos geradores de resíduos de saúde e determina que resíduos potencialmente infectantes não podem receber disposição final sem tratamento prévio que assegure a eliminação de suas características de patogenicidade. Essa Lei atribui aos serviços de saúde a responsabilidade pelo gerenciamento completo de seus resíduos, desde sua geração até a destinação e disposição final. Além disso, fixa que o importador, o fabricante e o distribuidor de medicamentos, bem como os prestadores de serviço de saúde são corresponsáveis pela coleta dos resíduos especiais resultantes dos produtos vencidos ou considerados, por decisão de autoridades competentes, inadequados ao consumo (BRASIL, 2010). 
A segregação do RSS por grupos é recomendada pela ANVISA e CONAMA e, se realizada, possibilitaria o melhor manejo e destino para cada tipo de resíduo, reduzindo custos e permitindo inclusive a implantação do sistema de coleta seletiva e reciclagem (SALES et al., 2009). Caberá aos estabelecimentos o gerenciamento de seus resíduos, desde a geração até a disposição final, de forma a atender aos requisitos ambientais e de saúde pública.

A classificação adotada pela ABNT, através da NBR 12.807/jun/13, que trata da terminologia do RSS, divide os resíduos em três classes: Classe A - Infectantes; Classe B - Especiais (químicos, farmacêuticos e radioativos) e Classe C - Comuns. Esses resíduos são gerados nas etapas de atendimento, diagnóstico e tratamento de pacientes em clínicas médicas, veterinárias, odontológicas, laboratórios, drogarias, centros de pesquisas (ABNT, 2013a).

A NBR no 12807 da ABNT define resíduo como "todo material desprovido de utilidade para o estabelecimento gerador" (ABNT, 2013a), em outras palavras, resíduo é todo material que foi utilizado em uma determinada situação e que não poderá ser utilizado novamente para a mesma finalidade, tornando sua vida útil não mais adequada, evidenciando o RSS.

Diante de toda a legislação vigente, é primordial que os hospitais implantem planos de gerenciamento de resíduos aprovados pelos órgãos fiscalizadores competentes, contemplando não apenas os fatores estéticos e de controle de infecção hospitalar, mas também considerando as questões ambientais tão importantes para a geração atual e futura.

É necessário desenvolver, através da educação, a consciência crítica dos grupos sociais, buscando o seu comprometimento com as questões ambientais, procurando alternativas para a equação desenvolvimento versus qualidade de vida.

No ano de 1993, ainda surge a NBR 12.808, que classifica os RSS quanto aos riscos potenciais ao trabalho, ao meio ambiente e à saúde pública, para que tenham gerenciamento adequado (ABNT, 1993a), conforme o quadro 5.

\begin{tabular}{|c|c|}
\hline \multicolumn{2}{|c|}{ CLASSIFICAÇÃO A - Resíduos infectantes } \\
\hline Tipo & Constituintes \\
\hline Tipo A.1 - Biológico & $\begin{array}{l}\text { Cultura, inóculo, mistura de microorganismos e meio de cultura } \\
\text { inoculado proveniente de laboratório clínico ou de pesquisa, vacina } \\
\text { vencida ou inutilizada, filtro de gases aspirados deáreas contaminadas } \\
\text { por agentes infectantes e resíduos contaminados por estes materiais. }\end{array}$ \\
\hline $\begin{array}{l}\text { Tipo A.2 - Sangue e } \\
\text { hemoderivados }\end{array}$ & $\begin{array}{l}\text { Bolsa de sangue após transfusão, com prazo de validade vencido ou } \\
\text { sorologia positiva, amostra de sangue para análise, soro, plasma e } \\
\text { outros subprodutos. }\end{array}$ \\
\hline $\begin{array}{l}\text { Tipo A.3 - Cirúrgico } \\
\text { Anatomopatológico e Exsudato }\end{array}$ & $\begin{array}{l}\text { Tecido, órgão, feto, peça anatômica, sangue e outros líquidos } \\
\text { orgânicos resultantes de cirurgia, necropsia e resíduos contaminados } \\
\text { por estes materiais. }\end{array}$ \\
\hline Tipo A.4 - Perfurante ou cortante & Agulha, ampola, pipeta, lâmina de bisturi e vidro. \\
\hline Tipo A.5 - Animal contaminado & $\begin{array}{l}\text { Carcaça ou parte de animal inoculado, exposto a microrganismos } \\
\text { patogênicos ou portador de doença infectocontagiosa, bem como } \\
\text { resíduos que tenham estado em contato com este. }\end{array}$ \\
\hline $\begin{array}{l}\text { Tipo A.6 - Assistência ao } \\
\text { paciente }\end{array}$ & $\begin{array}{l}\text { Secreções, excreções e demais líquidos orgânicos procedentes de } \\
\text { pacientes, bem como os resíduos contaminados por estes materiais, } \\
\text { inclusive restos de refeições. }\end{array}$ \\
\hline \multicolumn{2}{|r|}{ CLASSIFICAÇÃO B - Resíduos especiais } \\
\hline Tipo B.1 - Rejeito radioativo & $\begin{array}{l}\text { Material radioativo ou contaminado, com radionuclídeos } \\
\text { proveniente de laboratório de análises clínicas, serviços de medicina } \\
\text { nuclear e radioterapia. }\end{array}$ \\
\hline Tipo B.2 - Resíduo farmacêutico & Medicamentos vencidos, contaminados, interditado ou não utilizado. \\
\hline $\begin{array}{l}\text { Tipo B.3 - Resíduo químico } \\
\text { perigoso }\end{array}$ & $\begin{array}{l}\text { Resíduo tóxico, corrosivo, inflamável, explosivo, reativo genotóxico } \\
\text { ou mutagênico. }\end{array}$ \\
\hline Classe C - Resíduo comum & $\begin{array}{l}\text { Todos os que não se enquadram nos tipos A e B, com semelhança } \\
\text { aos resíduos domésticos que não oferecem risco adicional a saúde } \\
\text { pública. }\end{array}$ \\
\hline
\end{tabular}

Quadro 5 - Classificação dos RSS segundo NBR 12.808 (ABNT, 1993a). 
Para os autores Garcia e Zanetti-Ramos (2004), não se pode analisar a questão dos resíduos de serviços de saúde somente no aspecto da transmissão de doenças infecciosas, mas envolver também a questão da saúde do trabalhador e a preservação do meio ambiente. Acredita-se que o gerenciamento adequado dos resíduos possa contribuir significativamente para a redução da ocorrência de acidentes de trabalho.

De acordo com as considerações dos autores Silva e Hoppe (2005, p. 146)

Os RSS, apesar de representarem uma pequena parcela em relação ao total de resíduos gerados em uma comunidade, são fontes potenciais de propagação de doenças e apresentam um risco adicional aos trabalhadores dos serviços de saúde e a comunidade em geral, quando gerenciados de forma inadequada.

De acordo com os dados IBGE, cerca de 4.000 toneladas de resíduos produzidos pelos serviços de saúde são coletadas a cada dia, segundo as prefeituras de 5.507 municípios brasileiros. Quase todos esses produtos perigosos estão sendo despejados no ambiente sem o tratamento adequado - muitas vezes sem nenhum tratamento. Esses dados são demonstrados pelos resultados da Pesquisa Nacional de Saneamento Básico (PNSB), realizada pelo IBGE, nos quais os dados coletados relatam que, das prefeituras ouvidas no estudo, apenas $14 \%$ (779) disseram tratar o lixo de saúde adequadamente, seguindo o que determinavam as Resoluções $n^{\circ} 5 / 93$ e n ${ }^{\circ}$ 283/2001 do CONAMA (IBGE, 2010).

O tratamento adequado para resíduos de saúde pode ser considerado aquele que permita condições de segurança e eficiência e que possa modificar as características físicas, químicas e biológicas, ajustando-as a padrões aceitos legalmente como disposi- ção final. Nesse sentido, o tratamento pode ocorrer por si só ou associado a um tratamento prévio que impeça a disseminação dos agentes patogênicos ou outra forma de contaminação, acima do que legalmente é aceito. (PFITSCHER et al., 2007, p. 9).

Dessa forma, a questão dos RSS não pode ser analisada apenas no aspecto da transmissão de doenças infecciosas. A questão da saúde do trabalhador e a preservação do meio ambiente também estão envolvidas e são questões de preocupação da biossegurança.

Biossegurança é o conjunto de ações voltadas para a prevenção, minimização ou eliminação dos riscos inerentes às atividades de pesquisa, produção, ensino, desenvolvimento tecnológico e prestação de serviços. Esses riscos podem comprometer a saúde do homem e animais, o meio ambiente ou a qualidade dos trabalhos desenvolvidos (TEIXEIRA, 1996).

Em linhas gerais, são exigidos os seguintes acondicionamentos para alguns tipos de RSS: Resíduo infectante: recipiente (lixeira) com tampa e pedal, sinalizado com o símbolo internacional de risco biológico, forrado com saco plástico tipo II, regulamentado para resíduo infectante. Resíduos perfurocortantes: recipiente rígido, inquebrável, reforçado, estanque e sinalizado com o símbolo internacional de risco biológico. Resíduo comum: recipientes forrados com saco plástico do tipo I, regulamentado para resíduo comum.

A seguir um quadro (Quadro 6), com a classificação, acondicionamento, identificação, armazenamento temporário e disposição final dos RSS, instituídos a partir da RDC 306 (BRASIL, 2004), que dispõe sobre o regulamento técnico para o gerenciamento de resíduos de serviços de saúde. A resolução passou a considerar os riscos aos trabalhadores, à saúde e ao meio ambiente. 


\begin{tabular}{|c|c|}
\hline RESÍDUO - GRUPO & RDC - ANVISA 306 - TRATAMENTO \\
\hline \begin{tabular}{|c|} 
GRUPO A1 resíduo de laboratórios \\
de manipulação genética, meios de \\
cultura e materiais utilizados para o \\
preparo do mesmo, resíduos de fabri- \\
cação dos produtos biológicos, exceto \\
hemoderivados. \\
\end{tabular} & $\begin{array}{l}\text { Tratamento prévio na unidade geradora. } \\
\text { O acondicionamento em saco branco leitoso, se não houver } \\
\text { descaracterização física das estruturas, se houver descaracte- } \\
\text { rização acondicionados como resíduos do GRUPO D. }\end{array}$ \\
\hline $\begin{array}{c}\text { GRUPO A2 carcaças, peças anatômicas } \\
\text { e vísceras de animais submetidos a } \\
\text { processo de experimentação. }\end{array}$ & $\begin{array}{l}\text { Tratamento antes da disposição final. } \\
\text { Acondicionados em saco branco leitoso até } 2 / 3 \text { de sua capa- } \\
\text { cidade ou uma vez a cada } 24 \text { horas e devidamente identifica- } \\
\text { dos "PEÇAS ANATÔMICAS DE ANIMAIS". }\end{array}$ \\
\hline $\begin{array}{l}\text { GRUPO A3 Peças anatômicas e produ- } \\
\text { to de fecundação com peso menor que } \\
500 \text { gramas ou estatura menor que } 25 \\
\text { cm ou idade gestacional menor que } 20 \\
\text { semanas. }\end{array}$ & $\begin{array}{l}\text { Uma vez autorizado pelo órgão competente Município, Es- } \\
\text { tado ou Distrito Federal, realizar sepultamento em cemitério. } \\
\text { O tratamento é realizado por incineração ou cremação, com } \\
\text { equipamento devidamente licenciado para esse fim. } \\
\text { Acondicionados em saco vermelho até } 2 / 3 \text { de sua capacida- } \\
\text { de ou uma vez a cada } 24 \text { horas e devidamente identificados } \\
\text { "PEÇAS ANATÔMICAS". }\end{array}$ \\
\hline $\begin{array}{l}\text { GRUPO A4 Kits de linhas arteriais e } \\
\text { venosas e dialisadores descartados. } \\
\text { Filtros de ar e gases oriundos de áreas } \\
\text { críticas. }\end{array}$ & $\begin{array}{c}\text { Acondicionados em saco branco leitoso até } 2 / 3 \text { de sua capaci- } \\
\text { dade ou uma vez a cada } 24 \text { horas devidamente identificados. } \\
\text { Destino final sem tratamento prévio, em local licenciado } \\
\text { para este fim. }\end{array}$ \\
\hline \begin{tabular}{|l|} 
GRUPO A5 Materiais perfurocortantes \\
ou escarificantes, fluidos orgânicos, \\
tecidos, órgãos e outros materiais \\
resultantes da atenção a saúde de in- \\
divíduos desde que tenha suspeita ou \\
certeza de contaminação com príons. \\
\end{tabular} & $\begin{array}{l}\text { Acondicionados em dois sacos vermelhos até } 2 / 3 \text { de sua ca- } \\
\text { pacidade, sendo expressamente proibido o seu esvaziamento } \\
\text { e reaproveitamento. } \\
\text { Destino final sistema de incineração, definido na RDC AN- } \\
\text { VISA n }{ }^{\circ} 205 / 2002\end{array}$ \\
\hline
\end{tabular}

Quadro 6 - Classificação, tratamento e disposição final dos RSS segundo RDC 306 (BRASIL, 2004).

Dessa forma, torna-se importante frisar que em relação aos RSS - lixo hospitalar - apesar de representarem uma pequena parcela do total dos resíduos sólidos produzidos em uma comunidade, seu tratamento, acondicionamento e manuseio correto são particularmente importantes tanto para a segurança ocupacional dos funcionários, como para a saúde pública e qualidade do meio ambiente.

A educação ambiental é imprescindível para o desenvolvimento de projetos de saneamento ambiental. É sabido que a falta de saneamento básico pode provocar diversos riscos à saúde humana e de animais, bem como o comprometimento do meio ambiente.

\section{Conclusões}

Primeiramente há de se evidenciar a existência de normas e diretrizes públicas para buscar solucionar o descarte incorreto dos RSS, entretanto parece existir um distanciamento entre a teoria e o que é propriamente feito dentro e fora dos estabelecimentos de saúde, seja por parte dos gestores, profissionais de saúde e até mesmo pelos profissionais que manuseiam diariamente esses resíduos.

Diante da pesquisa, observou-se a importância de desenvolver instruções de trabalho para suprir a falta de informação, orientar e padronizar as operações que envolvem os RSS. Há uma necessidade de se estabelecer uma nova cultura de responsabilidade dos funcionários dos hospitais quanto a sua participação nos procedimentos sobre geração e manuseio de resíduos.

Para os órgãos que criam as Leis e juntamente os municípios, acredita-se haver uma necessidade de se ampliar as pesquisas na área de resíduos de serviços de saúde e espera-se que decisões legais sejam feitas com objetividade e sem a existência de brechas no que tange às responsabilidades do setor público e privado que atuam na coleta e disposição final dos RSS.

Portanto torna-se necessário um estudo da percepção pública de risco dos resíduos comuns e de serviços de saúde, e ainda a 
avaliação integrada de risco e gestão ambiental intra e extra-hospitalar dos resíduos de serviços de saúde.

Diante das afirmações e da literatura pesquisada, há de se conscientizar a população em geral sobre os riscos de doenças em que estão expostas ao entrar em contato com os RSS.

É importante frisar ainda que os RSS, na maioria das vezes, são armazenados adequadamente; entretanto, por falta de destino adequado, são jogados em locais com grande número de pessoas e animais, os conhecidos lixões. Os lixões, conforme estabelecidos em legislação brasileira, não são locais adequados ao descarte desse tipo de resíduo.

\section{Referências}

ASSOCIAÇÃO BRASILEIRA DE NORMAS TÉCNICAS - ABNT. NBR 12808: Resíduos de Serviços de Saúde: classificação. São Paulo, 1993a.

NBR 12810: Resíduos de Serviços de Saúde: especificação técnica transporte interno e externo:. São Paulo, 1993b.

. NBR 9190: Classifica sacos plásticos para acondicionamento de lixo. São Paulo, 1994.

. NBR 7500: Símbolos de risco e manuseio para o transporte e armazenamento. São Paulo, 2000.

. NBR 10.004: Classificação de resíduos sólidos: Rio de Janeiro, 2004.

. NBR 9191: Especificação de sacos plásticos para acondicionamento de lixo para coleta. São Paulo, 2008.

. NBR 12807: Resíduos de Serviços de Saúde: terminologia. São Paulo, 2013a.

NBR 12809: Resíduos de Serviços de Saúde: procedimentos necessários ao gerenciamento intraestabelecimento. São Paulo, 2013b.

ALMEIDA, M. de S. Elaboração de projeto, TCC, dissertação e tese: uma abordagem simples, prática e objetiva. São Paulo: Atlas, 2011.

ALVES, M. Como escrever teses e monografia: um roteiro passo a passo. Rio de Janeiro: Elsevier, 2007.

BRASIL. Ministério de Estado do Interior. Portaria MINTER $n^{\circ}$ 53, de $1^{\circ}$ de março de 1979. Disponível em: <http://www.ima.al.gov.br/legislacao/portariasministeriais/Portaria \%20nb0\%2053.79.pdf $>$. Acesso em: 20 maio 2013.

Presidência da República. Lei $n^{\circ} 6.938$, de 31 de agosto de 1981. Disponível em: < http:/ / www.planalto. gov.br/ccivil_03/leis/16938.htm>. Acesso em: 20 maio 2013.

. Ministério do Meio Ambiente. Conselho Nacional do Meio Ambiente (CONAMA). Resolução $n^{0}$ 6, de 19 de setembro de 1991. Disponível em: <http:/ / www. mma.gov.br/port/conama/res/res91/res0691.html>. Acesso em: 20 maio 2013.
Ministério do Meio Ambiente. Conselho Nacional do Meio Ambiente (CONAMA). Resolução $n^{\circ} 5$, de 5 de agosto de 1993. Disponível em: <http:/ / www. mma.gov.br/port/conama/legiabre.cfm?codlegi=130>. Acesso em: 20 maio 2013.

Ministério da Saúde. Secretaria Executiva. Projeto Reforço à reorganização do Sistema Único de Saúde (REFORSUS). Gerenciamento de resíduos de serviços de saúde. Brasília: Ministério da Saúde, 2001a.

Ministério do Meio Ambiente. Conselho Nacional do Meio Ambiente (CONAMA). Resolução $n^{\circ}$ 283, de 12 de julho de 2001b. Disponível em: <http:/ / www.mma.gov.br/port/conama/res/res01/res28301. html>. Acesso em: 20 maio 2013.

. Ministério da Saúde. Agência Nacional de Vigilância Sanitária. Resolução RDC $n^{\circ}$ 306, de 7 de dezembro de 2004. Disponível em: <http:/ / bvsms.saude.gov.br/ bvs/saudelegis/anvisa/2004/res0306_07_12_2004. html>. Acesso em: 20 maio 2013.

. Ministério do Meio Ambiente. Conselho Nacional do Meio Ambiente (CONAMA). Resolução $n^{\circ} 358$, de 29 de abril de 2005. Disponível em: <http:/ / www. mma.gov.br/port/conama/res/res05/res35805.pdf>. Acesso em: 20 maio 2013.

Presidência da República. Lei no 12.305, de 2 de agosto de 2010. Disponível em: <http:/ / www.planalto.gov.br/ccivil_03/_ato2007-2010/2010/lei/112305. htm>. Acesso em: 21 maio 2013.

CAMARGO, M. E. et al. Resíduos Sólidos de Serviço de Saúde: um estudo sobre o gerenciamento. Scientia Plena, v. 5, n. 7, p. 1-14, 2009.

CAMPONOGARA, S.; RAMOS, F. R. S.; KIRCHHOF, A. L. C. Um olhar sobre a interface trabalho hospitalar e os problemas ambientais. Rev. Gaúcha Enf., Porto Alegre, RS, v. 30, n. 4, p. 724-731, 2009.

CORRÊA, L. B.; LUNARDI, V. L.; CONTO, S. M. de. O processo de formação em saúde: o saber resíduos sólidos de serviços de saúde em vivência práticas. Rev. Bras. Enf., Brasília, DF, v. 60, n. 1, p. 21-25, jan./ fev. 2007.

CORRÊA, L. B.; LUNARDI, V. L.; SANTOS, S. S. C. Construção do saber sobre resíduos sólidos de serviços de saúde na formação em saúde. Rev. Gaúcha Enf., Porto Alegre, RS, v. 29, n. 4, p. 557-564, dez. 2008.

DOI, K. M.; MOURA, G. M. S. S. de. Resíduos sólidos de serviços de saúde: uma fotografia do comprometimento da equipe de enfermagem. Rev. Gaúcha Enf.; Porto Alegre, RS, v. 32, n. 2, p. 338-344, jun. 2011.

FERRAREZE, M. V. G. et al. Gerenciamento de resíduos de serviços de saúde: avaliação de um centro de terapia intensiva. REME - Rev. Min. Enf., Ribeirão Preto, SP, v. 9, n. 2, p. 133-139, abr./jun. 2005.

FERREIRA, J. A. Solid Waste and Nosocomial Waste: An Ethical Discussion. Cad. Saúde Pública, Rio de Janeiro, v. 11, n. 2, p. 314-320, apr./jun. 1995.

GARCIA, L. P.; ZANETTI-RAMOS, B. G. Gerenciamento dos resíduos de serviços de saúde: uma questão de biossegurança. Cad. Saúde Pública, Rio de Janeiro, v. 20, n. 3, p. 744-752, maio/jun. 2004.

INSTITUTO BRASILEIRO DE GEOGRAFIA E ESTATÍSTICA - IBGE. Pesquisa Nacional de Saneamento Básico 2008. Rio de Janeiro, 2010. 
MARTINS, G. de A.; LINTS, A. Guia para elaboração de monografias e trabalhos de conclusão de curso. São Paulo: Atlas, 2000.

NAIME, R.; RAMALHO, A. H. P.; NAIME, I. S. Avaliação do sistema de gestão dos resíduos sólidos do hospital de clínicas de Porto Alegre. Revista Espaço para a Saúde, Londrina, PR, v. 9, n. 1, p. 1-17, dez. 2008.

PFITSCHER, E. D. et al. A situação dos hospitais quanto ao gerenciamento dos aspectos e impactos ambientais. Cadernos EBAPE.BR, v. 5, n. 3, 2007.

RAMOS, Y.S. et al. Vulnerabilidade no manejo dos resíduos de serviço de saúde de João Pessoa (PB, Brasil). Ciências \& Saúde Coletiva, Rio de Janeiro, v. 16, n. 8, p. 3553-3560, ago. 2011.

RIBEIRO, C. da S. Análise das tecnologias de tratamento de resíduos biológicos de serviço de saúde em hospitais públicos no Município do Rio de Janeiro. 2008. 145f. Dissertação (Mestrado em Ciências na Área de Saúde Pública) Escola Nacional de Saúde Pública Sergio Arouca, Rio de Janeiro.

SALES, C. C. de L. et al. Gerenciamento dos resíduos sólidos dos serviços de saúde: aspectos do manejo interno no município de Marituba, Pará, Brasil. Ciência \&
Saúde Coletiva, Rio de Janeiro, v. 14, n. 6, p. 2231-2238, dez. 2009.

SCHNEIDER, V. E. et al. Manual de gerenciamento de resíduos sólidos em serviços de saúde. 2. ed. rev. e ampl. Caxias do Sul, RS: EDUCS, 2004.

SEVERINO, A. J. Metodologia do trabalho cientifico. São Paulo: Cortez, 2007.

SHINZATO, M. P. et al. Análise preliminar de riscos sobre o gerenciamento dos resíduos de serviços de saúde de uma instituição de ensino em Mato Grosso do Sul: um estudo de caso. Rev. Bras. Saúde Ocup., v. 35, n. 122, p. 340-352, 2010.

SILVA, C. E.; HOPPE, A. E. Diagnóstico dos Resíduos de Serviço de Saúde no interior do Rio Grande do Sul. Revista Engenharia Sanitária e Ambiental, v. 10, n. 2, p. 146-151, 2005.

TEIXEIRA, P. Biossegurança: uma questão multidisciplinar. Rio de Janeiro: Fiocruz, 1996.

VASCONCELLOS, E. A. de; et al. Diagnóstico do problema dos resíduos sólidos hospitalares: O caso de campina Grande (PB). HYGEIA - Revista Brasileira de Geografia Médica e da Saúde, v. 2, n. 3, p. 28-34. 2006. 